\title{
On the Topological Robustness of Vortex Modes at Microwave Frequencies
}

\author{
Mirko BARBUTO ${ }^{1}$, Andrea BASSOTTI ${ }^{1}$, Andrea ALU ${ }^{2}$, Filiberto BILOTTI ${ }^{3}$, Alessandro TOSCANO ${ }^{3}$ \\ ${ }^{1}$ Dept. of Engineering, Niccolò Cusano University, Via don Carlo Gnocchi 3, 00166 Rome, Italy \\ ${ }^{2}$ City University of New York, New York, NY 10031, USA \\ ${ }^{3}$ Dept. of Engineering, Roma Tre University, Via Vito Volterra 64, 00146 Rome, Italy \\ mirko.barbuto@unicusano.it
}

Submitted June 28, 2019 / Accepted June 29, 2019

\begin{abstract}
Vortex fields carrying orbital angular momentum are robust with respect to a wide range of external disturbances and exhibit self-healing properties. These peculiar characteristics have been deeply investigated at optical frequencies but only a few of them have been observed at microwaves. In this paper, we try to partially fill this gap by investigating the topological characteristics of vortex fields radiated by standard patch antennas. In particular, we describe the behavior of a vortex mode when a metallic screen is placed in the near field of the radiating patch. Through a proper set of full-wave numerical simulations, we show that the main characteristics of the vortex mode, i.e. the spiral phase profile and the amplitude null, are preserved even if the overall radiated field is strongly perturbed by the obstacle.
\end{abstract}

\section{Keywords}

Phase singularities, vortex modes, antenna radiation pattern

\section{Introduction}

Singularity points, i.e., points where a physical quantity assumes an undefined value, are ubiquitous in many branches of physics. Typical examples are phase singularities of optical vortices, which are optical fields characterized by an amplitude null in the center beam around which the phase circulates [1]. Their peculiar characteristics, strictly related to their topological nature, have been deeply investigated leading to a plethora of possible applications in imaging, communication, and identification systems, just to name a few. Generation of optical vortices is typically based on the interaction between light and matter. In particular, for imparting the required spiral phase front to a standard optical beam, spiral phase plates [2], pitch fork holograms [3] or Q-plates [4] have been exploited.

First investigated and experimentally realized at optical frequencies, vortex fields have been extended also to other frequency ranges. In particular, standard radiating structures, such as antenna arrays [5], parabolic or spiral reflectors [6], [7], and patch antennas [8] have been designed for radiating vortex modes at microwaves. In this frequency range, most research efforts have been focused on exploiting the intrinsic orthogonality of different vortex modes in communication systems. However, despite the first enthusiastic proclamations, multiplexing systems based on vortex modes turned out to be a sub-case of MIMO systems with, consequently, no particular advantages compared to other standard multiplexing techniques. Nevertheless, investigations of microwave vortices have led to other several interesting findings such as the possibility to be exploited for identification systems [9], for achieving anomalous polarization states [10], or for shaping the antenna radiation pattern [11]. Some of these findings are related to the robustness of phase singularity points that, being a topological characteristic of vortex modes, cannot be easily suppressed [12]. In particular, we have exploited this property to manipulate the overall radiation pattern of a patch antenna generating vortex modes [11]. In fact, by designing an antenna able to radiate simultaneously a vortex and a vortex-free mode, the latter cannot suppress the phase singularity point of the total radiated beam, which, however, can be simply moved by acting on the amplitudes and phases of excitation of the two constituting beams. As phase singularity points are necessarily coexistent with amplitude nulls, this allows to directly manipulate the overall radiation pattern.

We remark here that this property is not the only form of robustness exhibited by vortex modes. In fact, as demonstrated at optical frequencies, this kind of electromagnetic fields is robust with respect to a wide range of external perturbations and, in particular, shows self-healing properties when part of the beam itself is obstructed by an opaque obstacle [13]. However, to the best of our knowledge, this behavior has been not exploited or investigated at lower frequencies.

The main purpose of this contribution is, thus, to extend to microwave frequencies some results about the selfreconstruction capabilities of optical vortex beams. In par- 
ticular, we study the behavior of a vortex mode radiated by a patch antenna when a metallic screen is placed in the near field of the antenna itself. Through a proper set of fullwave numerical simulations, we will show that the peculiar properties of the radiated field and, thus, of the antenna radiation pattern, persist also when the near field of the antenna itself is strongly perturbed.

\section{Description of the Simulation Setup}

After the first experimental realization at microwaves, several standard radiating systems have been modified to support electromagnetic radiation with a phase singularity. One of the simpler solutions is the use of a single patch antenna properly designed to radiate circularly polarized (CP) higher order modes. In fact, as shown in [14], a CP $\mathrm{TM}_{n 1}$ mode exhibits a phase singularity of order $n-1$ in the center beam. Therefore, to generate a vortex mode of the first order, we use an elliptical patch antenna excited with a single coaxial feed, as shown in Fig. 1. In this way, we can properly choose the dimensions of the two main axis of the elliptic patch, $A_{1}$ and $A_{2}$, to excite two degenerate and in quadrature $\mathrm{TM}_{21}$ modes and, thus, to radiate an overall $\mathrm{CP} \mathrm{TM}_{21}$ mode around the center operating frequency $(2.4 \mathrm{GHz})$. Please note that more details on the design procedure can be found in [14].

In order to investigate the robustness of the radiated vortex mode, we have placed in front of the patch antenna opaque obstacles of different dimensions and shapes. However, for sake of brevity and for comparing the results with the ones already reported in optics [13], in this paper we focus our attention on the case of a thin metallic screen placed parallel to the patch antenna, as shown in Fig. 1.

The patch (with dimensions: $A_{1}=75.2 \mathrm{~mm}, A_{2}=$ $81.3 \mathrm{~mm}$ ) is etched on a Rogers Duroid ${ }^{\mathrm{TM}}$ RT5870 dielectric substrate $(0.787 \mathrm{~mm}$ thick $)$, while the screen overall dimensions are $120 \times 94 \times 1 \mathrm{~mm}^{3}$. In this way, the metallic screen can intercept most of the field radiated by the patch, including the singularity point that lies in the broadside direction. Moreover, different screen positions $d$ have been

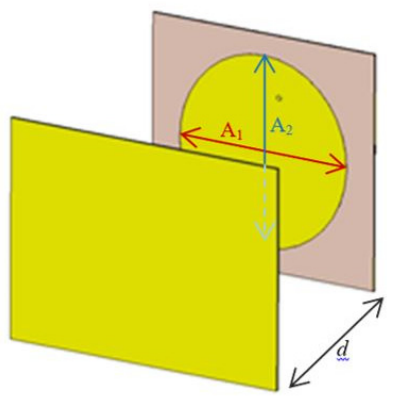

(a)

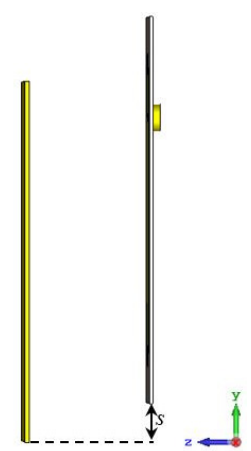

(b)
Fig. 1. Perspective (a) and lateral (b) view of the simulation setup consisting of an elliptical patch antenna generating a vortex mode of the first order and a metallic screen placed in its close proximity. considered, as well as different vertical shifts $s$ of the screen with respect to the lower edge of the patch.

\section{Simulation Results}

First, in order to verify that the obstacle does not compromise the effectiveness of the antenna in radiating the selected vortex mode, we have evaluated the matching performance of the obstructed antenna for different screen distances and for a fixed shift with respect to the lower edge of the patch $(s=-10 \mathrm{~mm})$. As expected, by approaching the screen to the antenna plane, the matching properties worsen. However, for all the cases of interest, a good impedance matching has been obtained at the center operating frequency, as shown in Fig. 2. Moreover, from this figure we can observe that the reflection coefficient magnitude exhibits two partially overlapped resonant frequencies, corresponding to the two degenerated $\mathrm{TM}_{21}$ modes required to obtain a circular polarization [14].

Then, in order to evaluate the effect of the obstacle on the topological properties of the vortex mode, we have analyzed the field radiated by the patch antenna for different screen positions $(d=\lambda / 4, \lambda / 2,3 \lambda / 4$ and $\lambda$; where $\lambda$ is the operational wavelength at the center operating frequency). As can be appreciated in Fig. 3, the obstacle strongly perturbs the radiated field that, on the plane of the screen, does not exhibit the central phase singularity point that characterizes any vortex mode. However, this does not compromise the presence of the spiral phase front. In fact, moving away from the screen, the phase singularity point regenerates itself, as shown in Fig. 4, confirming the self-healing properties of vortex modes.

Please note, however, that the obstacle affects the overall field by moving the phase singularity point with respect to the original center position. This effect is equivalent to the one already investigated at microwaves [10], where a properly superimposed vortex-free mode was used to control the phase singularity point of a vortex mode of the first order. Therefore, we can infer that the presence of the obstacle gives rise to an undesired vortex-free com-

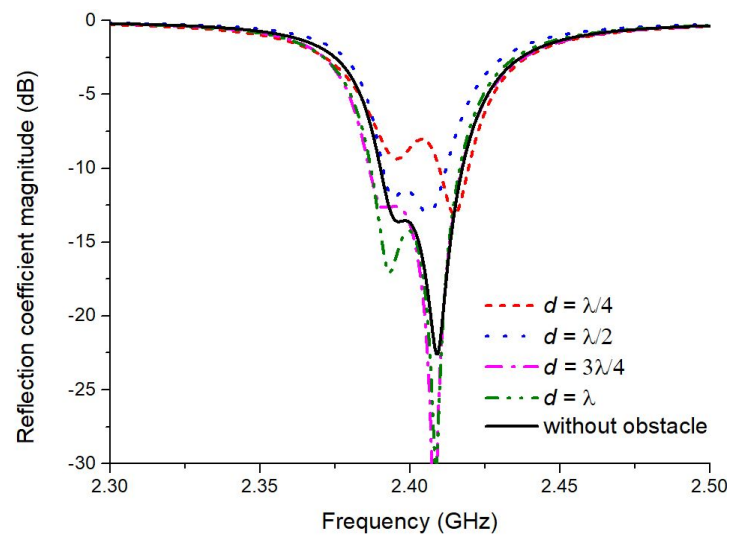

Fig. 2. Reflection coefficient magnitude of the antenna shown in Fig. 1 for different screen positions. 
ponent that, as demonstrated in [10], has a direct impact also on the radiation pattern of the overall structure. In fact, the shift of the phase singularity points along the vertical direction induces a tilt of the overall radiation pattern, as shown in Fig. 5. In particular, the deflection of the radiation null toward the obstacle decreases as the distance between the obstacle and antenna increases.

Please note that we have reported here only the phase patterns of the $x$-component of the radiated electric field but, working with circular polarized modes, a similar behavior can be observed also for the $y$-component.

Finally, we have also evaluated, for a given distance of the screen $(d=\lambda / 2)$ and without loss of generality, the effect of a vertical shift of the screen itself. Again, the

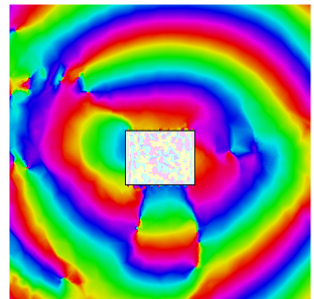

(a)

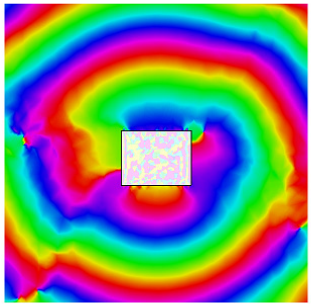

(c)

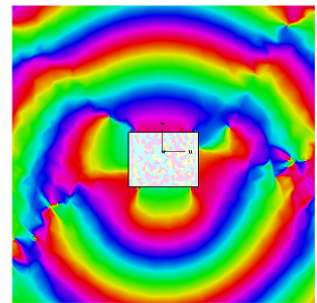

(b)

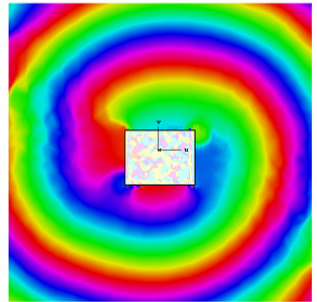

(d)

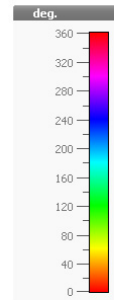

Fig. 3. Phase patterns of the electric field (x-component) radiated by the antenna reported in Fig. 1 on the screen plane: (a) $d=\lambda / 4$, (b) $d=\lambda / 2$, (c) $d=3 \lambda / 4$, (d) $d=\lambda$.

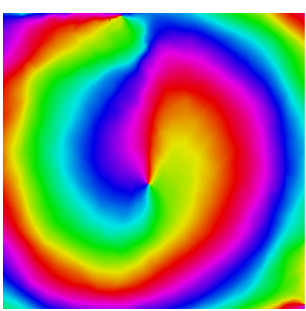

(a)

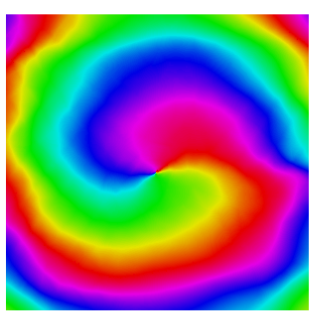

(c)



(b)

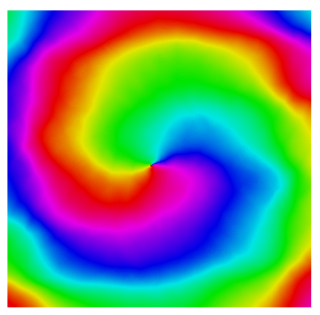

(d)



Fig. 4. Phase patterns of the electric field ( $x$-component) radiated by the antenna reported in Fig. 1 at a distance from the screen equal to $2 \lambda$ : (a) $d=\lambda / 4$, (b) $d=\lambda / 2$, (c) $d=3 \lambda / 4$, (d) $d=\lambda$. reflection coefficient magnitude, reported in Fig. 6, confirms the good impedance matching of the antenna in all the considered scenarios. Moreover, by acting on the vertical position of the screen, we change the portion of the radiated field intercepted by the obstacle. As shown in Fig. 7, this results in a null position that increasingly moves away from the broadside direction as the vertical displacement of the screen is increased. Please note also that the radiation null is tilted upward or downward depending on whether the major part of the screen is placed above or below the central point of the patch.

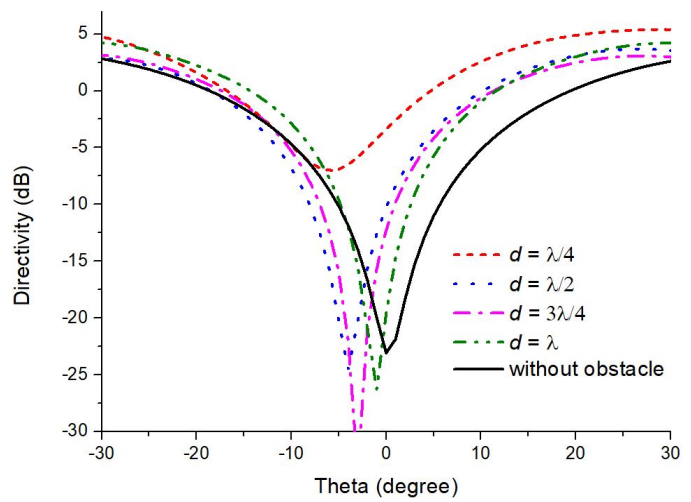

Fig. 5. Directivity pattern on the $y z$-plane of the antenna reported in Fig. 1.

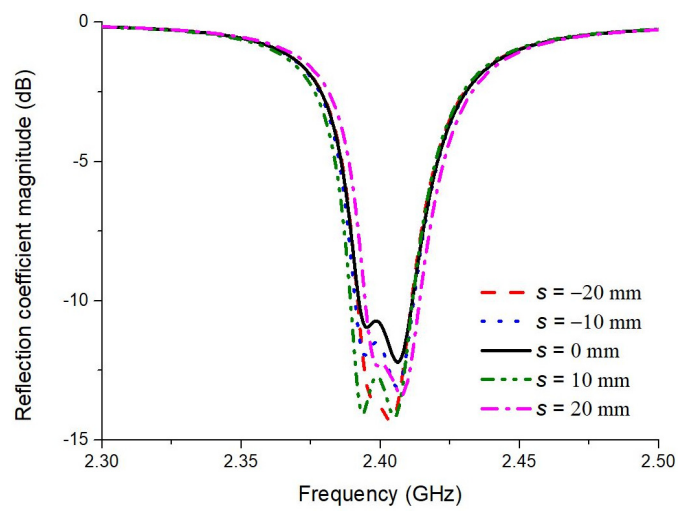

Fig. 6. Reflection coefficient magnitude of the antenna shown in Fig. 1 for different screen shift along the vertical direction.



Fig. 7. Directivity pattern on the $y z$-plane of the antenna reported in Fig. 1 for different screen shift along the vertical direction. 
We remark here that these results do not exhaustively investigate the problem of a metallic screen that alters the amplitude and phase distributions of a vortex mode radiated by a patch antenna. In fact, the effects of the shape and dimensions of the obstacle should be further investigated. However, our preliminary analysis suggests that topological robustness of vortex modes may be exploited for designing complex antenna systems, whose radiation characteristics are only slightly perturbed by the presence of obstacles in their near field.

\section{Conclusions}

In this paper, we have investigated the self-healing properties of vortex modes generated at microwaves. For this purpose, we have analyzed a simple simulation setup consisting of a circular patch antenna (properly designed to radiate a vortex mode with a phase singularity of the first order) and a thin metallic screen placed in its close proximity. By studying the field distributions beyond the screen, we have observed that the phase singularity point of the vortex mode actually persists even in the presence of strong disturbances introduced by the screen. This has also an effect on the overall radiation pattern that, despite the presence of a large metallic object in the near field of the antenna, still exhibits a deep radiation null. These findings can be exploited to predict and mitigate unwanted effects of opaque objects placed in the near field of existing radiating structures. Moreover, they suggest that topological properties of vortex modes could be used for designing new antenna systems with robust radiation characteristics with respect to large obstacles placed in their close proximity.

\section{References}

[1] YAO, A. M., PADGETT, M. J. Orbital angular momentum: origins, behavior and applications. Advances in Optics and Photonics, 2011, vol. 3, p. 161-204. DOI: 10.1364/AOP.3.000161

[2] BEIJERSBERGEN, M. W., COERWINKEL, R. P. C., KRISTENSEN, M., et al. Helical-wavefront laser beams produced with a spiral phase plate. Optics Communications, 1994, vol. 112, no. 5-6, p. 321-327. DOI: 10.1016/0030-4018(94)90638-6

[3] BAZHENOV, V. Y., VASNETSOV, M. V., SOSKIN, M. S. Laser beams with screw dislocations in their wavefronts. Journal of Experimental and Theoretical Physics Letters, 1990, vol. 52, no. 8, p. $429-431$.

[4] HEMSING, E., KNYAZIK, A., DUNNING, M., et al. Coherent optical vortices from relativistic electron beams. Nature Physics, 2013, vol. 9, p. 549-553. DOI: 10.1038/nphys 2712

[5] THIDÉ, B., THEN, H., SJOHOLM, J., et al. Utilization of photon orbital angular momentum in the low-frequency radio domain. Physical Review Letters, 2007, vol. 99, no. 8, p. 1-4. DOI: 10.1103/PhysRevLett.99.087701

[6] TAMBURINI, F., MARI, E., SPONSELli, A., et al. Encoding many channels on the same frequency through radio vorticity: first experimental test. New Journal of Physics, 2012, vol. 14, p. 1-17. DOI: $10.1088 / 1367-2630 / 14 / 3 / 033001$
[7] TAMBURINI, F. MARI, E., THIDE, B., et al. Experimental verification of photon angular moment and vorticity with radio techniques. Applied Physics Letters, 2011, vol. 99, no. 20, p. 204102-1-204102-3. DOI: 10.1063/1.3659466

[8] BARBUTO, M., TOSCANO, A. BILOTTI, F. Single patch antenna generating electromagnetic field with orbital angular momentum. In 2013 IEEE Antennas and Propagation Society International Symposium (APSURSI). Orlando (FL, USA), 2013, p. 1866-1867. DOI: 10.1109/APS.2013.6711591

[9] LIN, M., GAO, Y., LIU, P., et al. Super-resolution orbital angular momentum based radar targets detection. Electronics Letters, 2016, vol. 52, no. 13, p. 1168-1170. DOI: 10.1049/el.2016.0237

[10] BARBUTO, M., BILOTTI, F., TOSCANO, A. Patch antenna generating structured fields with a Mobius polarization state. IEEE Antennas and Wireless Propagation Letters, 2017, vol. 16, p. 1345-1348. DOI: 10.1109/LAWP.2016.2634081

[11] BARBUTO, M., MIRI, M.-A., ALÙ, A., et al. Exploiting the topological robustness of composite vortices in radiation systems. Progress In Electromagnetics Research, 2018, vol. 162, p. 39-50. DOI: 10.2528 /PIER18033006

[12] GalveZ, E. J., SMILEY, N., FERnANDES, N. Composite optical vortices formed by collinear Laguerre-Gauss beams. Proceedings of SPIE 6131, Nanomanipulation with Light II, 613105, Feb. 9, 2006. DOI: 10.1117/12.646074

[13] VASNETSOV, M. V., MARIENKO, I. G., SOSKIN, M. S. Selfreconstruction of an optical vortex. Journal of Experimental and Theoretical Physics Letters, 2000, vol. 71, no. 4, p. 130-133. DOI: $10.1134 / 1.568297$

[14] BARBUTO, M., TROTTA, F., BILOTTI, F., et al. Circular polarized patch antenna generating orbital angular momentum. Progress In Electromagnetics Research, 2014, vol. 148, p. 23-30. DOI: 10.2528/PIER14050204

\section{About the Authors ...}

Mirko BARBUTO was born in Rome, Italy, on April 26, 1986. He received the B.S., M.S. and Ph.D. degrees from "Roma Tre" University, Rome, Italy, in 2008, 2010 and 2015, respectively. Since September 2013, he is with the "Niccolò Cusano" University, Rome, Italy, where he works as an Assistant Professor of electromagnetic field theory. His main research interests are in the framework of applied electromagnetics, with an emphasis on antennas and components at RF and microwaves, cloaking devices for radiating systems, metamaterials, electromagnetic structures loaded with non-linear or non-foster circuits, topological properties of vortex fields and smart antennas for GNSS technology.

He is a member of the Technical Program Committee of the International Congress on Artificial Materials for Novel Wave Phenomena (since 2017) and he serves as a Technical Reviewer of the major international conferences and journals related to electromagnetic field theory and metamaterials. He has been recipient of the Outstanding Reviewers Award assigned by the Editorial Board of the IEEE Transactions on Antennas \& Propagation for four consecutive years (2015-2018) and he has received the same award by the Editorial Board of the IEEE Antennas and Wireless Propagation Letters for two consecutive years 
(2017-2018). In 2017 he has been selected as one of the Best Reviewers by the Editorial Board of Radioengineering Journal. Since 2015, he is the Proceeding Editor for the annual International Congress on Engineered Material Platforms for Novel Wave Phenomena - Metamaterials.

Dr. Barbuto is currently member of the Italian Society on Electromagnetics (SIEM), of the National Inter-University Consortium for Telecommunications (CNIT), of the Virtual Institute for Artificial Electromagnetic Materials and Metamaterials (Metamorphose VI AISBL), and of the Institute of Electrical and Electronics Engineers (IEEE). Currently, he is the author of more than 50 papers in international journals and conference proceedings.

Andrea BASSOTTI was born in Rome, Italy, on October 1, 1990. He received the B.S. degree from "Niccolò $\mathrm{Cu}-$ sano" University, Rome, Italy, in 2018 and he is currently pursuing a M.Sc. degree from the same university. His research interests include the generation of vortex fields at microwave frequencies and the exploitation of their topological properties to design robust antenna systems.

Andrea ALÙ received the Laurea, M.S., and Ph.D. degrees from the University of Roma Tre, Rome, Italy, in 2001, 2003 and 2007, respectively.

From 2002 to 2008, he has been periodically with the University of Pennsylvania (UPenn), Philadelphia, PA, USA, where he has also developed significant parts of his Ph.D. and postgraduate research. After spending one year as a Post-Doctoral Research Fellow at UPenn in 2009, he joined the faculty of The University of Texas at Austin, Austin, TX, USA, where he was the Temple Foundation Endowed Professor until 2018. He is currently an Adjunct Professor, a Senior Research Scientist, and a member of the Wireless Networking and Communications Group, at The University of Texas at Austin. He is currently the Founding Director of the Photonics Initiative with the Advanced Science Research Center, Graduate Center of the City University of New York (CUNY), New York, NY, USA. He is also the Einstein Professor of physics with the CUNY Graduate Center, New York, NY, USA, and a Professor of electrical engineering with the City College of New York, New York. He has co-authored an edited book Optical Antennas, over 400 journal papers, and over 30 book chapters, with over 20000 citations to date. His current research interests include metamaterials and plasmonics; electromangetics, optics, and nanophotonics; acoustics, scattering, nanocircuits, and nanostructures; miniaturized antennas and nanoantennas; and RF antennas and circuits.

Dr. Alù is a Full Member of the International Union of Radio Science (URSI) and a fellow of the Optical Society of America (OSA), the International Society for Optics and Photonics (SPIE), the Institute of Electrical and Electronic Engineers (IEEE) and the American Physical Society. He has received several research awards, including the URSI Commission B in 2004, 2007, and 2010, the Young Scientist Awards from URSI General Assembly in 2005, the AFOSR and the DTRA Young Investigator Awards in
2010 and 2011, the NSF CAREER Award in 2010, the URSI Issac Koga Gold Medal in 2011, the SPIE Early Career Investigator Award in 2012, the Franco Strazzabosco Award for Young Engineers in 2013, the IUPAP Young Scientist Prize in Optics in 2013, the OSA Adolph Lomb Medal in 2013, the IEEE Microwave Theory and Techniques (MTT) Outstanding Young Engineer Award in 2014, the NSF Alan T. Waterman Award in 2015, the ICO Prize in Optics in 2016, the Inaugural MDPI Materials Young Investigator Award in 2016, the Kavli Foundation Early Career Lectureship in Materials Science in 2016, the Inaugural ACS Photonics Young Investigator Award Lectureship in 2016, the Edith and Peter O'Donnell Award in Engineering in 2016, the Vannevar Bush Faculty Fellowship from the Department of Defense in 2019, and the IEEE Kiyo Tomiyasu Award in 2019. His students, Y. Zhao in 2011 and J. Soric in 2012, have also received several awards, including student paper awards at the IEEE Antennas and Propagation Symposia. He was the Technical Program Chair of the IEEE Antennas and Propagation (AP)-S Symposium in 2016 and the International Metamaterials Conference in 2014 and 2015. He has organized and chaired various special sessions in international symposia and conferences. He is currently on the Editorial Boards of Physical Review B, the New Journal of Physics, Advanced Optical Materials, MDPI Materials, EPJ Applied Metamaterials, and ISTE Metamaterials. He served as an Associate Editor for the IEEE Antennas and Wireless Propagation Letters, Scientific Reports, Metamaterials, Advanced Electromagnetics, and Optics Express. He has guest edited special issues for the IEEE Journal of Selected Topics in Quantum Electronics, the IEEE Antennas and Wireless Propagation Letters, Nanophotonics, the Journal of Optics, the Journal of the Optical Society of America B, Photonics and Nanostructures: Fundamentals and Applications, Optics Communications, Metamaterials, and Sensors on a variety of topics involving metamaterials, plasmonics, optics, and electromagnetic theory. He has been a Simons Investigator in physics since 2016, has been selected twice as the finalist of the Blavatnik Award for Young Scientists in 2016, 2017, 2018 and 2019, and has been a Highly Cited Researcher from Web of Science since 2017. He has been serving as an OSA Traveling Lecturer since 2010, the IEEE AP-S Distinguished Lecturer since 2014, and as the IEEE joint AP-S and MTT-S Chapter for Central Texas.

Filiberto BILOTTI received the Laurea and Ph.D. degrees in Electronic Engineering from Roma Tre University, Rome, Italy, in 1998 and 2002, respectively.

Since 2002, he has been with the Department of Engineering, Roma Tre University, where he is a Full Professor of electromagnetic field theory since 2014 and also the Director of the Antennas and Metamaterials Research Laboratory since 2012 . He has authored over 470 papers in international journals, conference proceedings, and book chapters. His current research interests include microwave and optical applications of artificial electromagnetic materials, metamaterials, and metasurfaces. 
Dr. Bilotti served as a member of the Editorial Boards of the International Journal on RF and Microwave ComputerAided Engineering from 2009 to 2015 and Scientific Reports (Nature) from 2013 to 2016. He was an elected member of the Board of Directors from 2007 to 2013. He has been serving as a member of the Editorial Board of EPJ Applied Metamaterials since 2013. He was a recipient of the Raj Mittra Travel Grant Senior Researcher Award in 2007 and Finmeccanica Group Innovation Award in 2014. He served as the Chairman of the Steering Committee and has been elected the General Chair of the First International Congress on Advanced Electromagnetic Materials and Metamaterials in Microwaves and Optics-Metamaterials from 2008 to 2014 and from 2015 to 2018, respectively. He also served as a member of the Technical Program, Steering, and Organizing Committee of several national and international conferences, as an Organizer and Chairman of special sessions focused on the applications of metamaterials at microwave and optical frequencies, and as an Associate Editor for Metamaterials from 2007 to 2013 and the IEEE Transactions on Antennas and Propagation from 2013 to 2017. He organized the First International Congress on Advanced Electromagnetic Materials and Metamaterials in Microwaves and Optics-Metamaterials 2007 in Rome in 2007. He served as a technical reviewer for the major international journals related to electromagnetic field theory and metamaterials. He was the President of the Virtual Institute for Artificial Electromagnetic Materials and Metamaterials (METAMORPHOSE VI, the International Metamaterials Society) from 2013 to 2019.

Alessandro TOSCANO (Capua, 1964) graduated in Electronic Engineering from Sapienza University of Rome in 1988 and he received his $\mathrm{PhD}$ in 1993. Since 2011, he has been a Full Professor of Electromagnetic Fields at the Engineering Department of Roma Tre University. He carries out an intense academic and scientific activity, both nationally and internationally.

From April 2013 to January 2018 he was a member of Roma Tre University Academic Senate. From October 2016 to October 2018, he was a member of the National Commission which enables National Scientific Qualifications to Full and Associate Professors in the tender sector
09/F1 - Electromagnetic fields. Since 23rd January 2018 he has been a Vice-Rector for Innovation and Technology Transfer.

In addition to his commitment in organizing scientific events, he also carries out an intense editorial activity as a member of the review committees of major international journals and conferences in the field of applied electromagnetics. He has held numerous invited lectures at universities, public and private research institutions, national and international companies on the subject of artificial electromagnetic materials, metamaterials and their applications. He actively participated in founding the international association on metamaterials Virtual Institute for Advanced Electromagnetic Materials - METAMORPHOSE, VI. He coordinates and participates in several research projects and contracts funded by national and international public and private research institutions and industries.

Alessandro Toscano's scientific research has as ultimate objective the conceiving, designing and manufacturing of innovative electromagnetic components with a high technological content that show enhanced performance compared to those obtained with traditional technologies and that respond to the need for environment and human health protection. His research activities are focused on three fields: metamaterials and unconventional materials, in collaboration with Professor A. Alù's group at The University of Texas at Austin, USA, research and development of electromagnetic cloaking devices and their applications (First place winner of the Leonardo Group Innovation Award for the research project entitled: 'Metamaterials and electromagnetic invisibility') and the research and manufacturing of innovative antenna systems and miniaturized components (first place winner of the Leonardo Group Innovation Award for the research project entitled: "Use of metamaterials for miniaturization of components" - MiniMETRIS).

$\mathrm{He}$ is the author of more than one hundred publications in international journals indexed ISI or Scopus; of these on a worldwide scale, three are in the first 0.1 percentile, five in the first 1 percentile and twenty-five in the first 5 percentile in terms of number of quotations and journal quality. 